\title{
A comparison of the different 3D CT scanning modes on the GTV delineation for the solitary pulmonary lesion
}

\author{
Dong-ping Shang ${ }^{1}$, Cheng-xin Liu ${ }^{1}$ and Yong Yin ${ }^{1,2^{*}}$
}

\begin{abstract}
Objectives: To investigate the impacts of the different three-dimensional CT (3DCT) scanning modes on the GTV delineation for solitary pulmonary lesion (SPL) based on four-dimensional $\mathrm{CT}(4 \mathrm{DCT})$, and to evaluate the feasibility of using the spiral CT scan in CT simulation.

Materials and methods: Twenty-one patients with SPL underwent axial CT scan, spiral CT scan and 4DCT simulation scan during free-breathing, respectively. The same clinical radiation oncologist delineated the gross tumor volume (GTV) under the same CT window setting. GTV $\mathrm{A}_{\mathrm{A}}$ and $\mathrm{GTV}_{\mathrm{S}}$ were created from the axial and spiral images, respectively. ITV $\mathrm{MIP}_{\mathrm{P}}$ was created from the maximum intensity projection (MIP) reconstructed 4D images. The target volumes and position between $\mathrm{GTV}_{\mathrm{A}} \mathrm{GTV}_{\mathrm{S}}$ and ITV $\mathrm{TIP}_{\mathrm{P}}$ were compared. The matching index (MI) between $\mathrm{GTV}_{\mathrm{A}}$ and $\mathrm{GTV} \mathrm{V}_{\mathrm{S}}$, and the correlation between $\mathrm{Ml}$ and $\mathrm{GTV}_{\mathrm{S}}$ were evaluated.

Results: ITV MIP was significantly larger than $\operatorname{GTV}_{\mathrm{A}}$ and $\operatorname{GTV}_{S}\left(p_{S}=0.000\right)$. The ratios of ITV $\mathrm{TIP}_{\mathrm{P}}$ to $\mathrm{GTV}_{\mathrm{A}}$ and $\mathrm{GTV}_{S}$ were $1.57 \pm 0.54$ and $1.66 \pm 0.61$, respectively. There was no significant difference between $\operatorname{GTV}_{A}$ and $\operatorname{GTV}_{S}(p=0.16)$. A comparison of the centroidal positions in $x, y$, and $z$ directions for $G T_{A}, G T V_{S}$ and $G T V_{4 D m i p}$ showed no significant difference $\left(p_{\mathrm{x}}=0.17, p_{\mathrm{y}}=0.40, p_{z}=0.48\right)$. Additionally, there was no difference between distances from the centroidal positions of $\mathrm{GTV}_{\mathrm{A}}$ and $\mathrm{GTV}_{\mathrm{S}}$ to the origin of coordinates $(p=0.51)$. Ml between $\mathrm{GTV}_{\mathrm{A}}$ and $\mathrm{GTV}_{\mathrm{S}}$ was 0.41 \pm 0.24 (range 0-0.89), and it was positively correlated with the tumor volume $(r=0.64, p=0.002)$.

Conclusion: There was no impact on the volume or centroidal position of GTV by the axial scan or spiral scan in 3DCT simulation for SPL. MI between $\mathrm{GTV}_{\mathrm{A}}$ and $\mathrm{GTV}_{\mathrm{S}}$ was small. A positively correlation was found between $\mathrm{Ml}$ and $\mathrm{GTV}_{\mathrm{S}}$. Relative to axial scanning mode, spiral $\mathrm{CT}$ scan was more timesaving and more efficient, it was feasible in 3DCT simulation for SPL.
\end{abstract}

Keywords: CT scan, Solitary pulmonary lesion, Gross tumor volume

\section{Introduction}

The aim of radiation treatment is to increase the radiotherapy effects on the tumor while avoiding excessive toxicity $[1,2]$. Computed tomography (CT) simulation and accurately determining the target margin were prerequisite for the successful oncology radiotherapy. Three-dimensional CT (3DCT) and four-dimensional CT (4DCT) simulation techniques are both in used, and 4DCT simulation is currently the leading simulation technology. It has enabled CT

\footnotetext{
*Correspondence: yongyinsd@163.com
'Department of Radiation Oncology, Shandong Tumor Hospital, Shandong

*Correspondence: yongyinsd@163.com
'Department of Radiation Oncology, Shandong Tumor Hospital, Shandong Province 250117, China Department of Radiation Oncology, Shandong Tumor Hospital, 440 Jiyan
Road, Jinan 250117, China ${ }^{2}$ Department of Radiation
Road, Jinan 250117, China
}

data acquisition to be correlated to the respiratory cycle(s), and allows a series of 3DCT data sets to be acquired at a number of points in a patient's breathing cycle offering visualization of tumor motion on a patient by basis [3-5]. However, the 3DCT simulation is still widely used for 3D conformal radiotherapy (3D-CRT) at present. There were two scanning modes in 3DCT simulation, which were axial and spiral scanning mode. Because the speed of tube rotation in axial mode was slower than that in spiral mode, we often treat head and neck tumors that are not affected by respiratory motion with spiral scan [6]. For thoracic and abdominal tumor, respiratory motion and heartbeat are important factors to determine the gross tumor volume 
(GTV). The slow speed scanning mode could collect more respiratory motion information, so we usually adopted axial scanning mode for simulation during free-breathing [7]. But whether the axial scan and spiral scan that are usually adopted would influence the GTV of the lung tumor in motion during free-breathing was unclear. Basing on 4DCT technique, this study evaluates how the axial and spiral scanning modes affect the position, volume and spatial match relationships on the GTV delineation for the solitary pulmonary lesion (SPL) in 3DCT simulation.

\section{Materials and methods \\ Patients}

From March 2010 to September 2012, 21 patients with pathologically proven peripheral lung cancer or pulmonary metastasis who were treated with stereotactic body radiotherapy (SBRT) at Shandong Tumor Hospital were included in this study. Institutional Review Board of the hospital approval and informed consent were obtained for the present study. All patients had SPL with no adhesion between the tumor edge and pleura. And all of them could breathe freely and cooperate with CT scanning. The subjects consisted of 13 males and 8 females. The age of the patients ranged from 38 to 78 years, with a median age of 59 years. The lesion volumes ranged from $1.45 \mathrm{~cm}^{3}$ to $35.41 \mathrm{~cm}^{3}$, located in left lung in 9 cases and right lung in 12 cases, respectively.

\section{CT simulation and image acquisition}

The patients were immobilized in the supine position with their arms raised above head using vacuum bags. First, the axial scan was performed during free-breathing using Philip Brilliance big bore CT scanner (Philips Medical Systems, Inc., Cleveland, OH, USA). And then patients were conducted spiral scan and 4D scan consecutively, the image layer thickness was set to $3 \mathrm{~mm}$. The axial scan cycle (scan + in-couch time) was $2.8 \mathrm{~s}$. The pitch was 0.938 under spiral scanning. 4D technique was a kind of slow spiral scan mode, which would adjust the pitch according to respiratory rate in $4 \mathrm{D}$ scanning (pitch: $0.09 \sim 0.15$ ) [8]. The respiratory signal was recorded with the Real-Time Position Management (RPM, Varian Medical Systems, Palo Alto, CA, USA) by tracking the trajectory of the infrared markers placed on the patient's abdomen [9]. The respiratory signal was transferred to the CT scanning device, where the respiratory phase was matched with the acquired image(s). The $4 \mathrm{D}$ volume data were reconstructed into ten sequence images of different respiration phase $(0 \%, 10 \%, 20 \%$...90\%) according to the respiration signal acquired by the RPM system, the $0 \%$ phase corresponded to the end-inhalation and the $50 \%$ phase corresponded to the end-exhalation. The ten sequence images were reconstructed with the same thickness of $3 \mathrm{~mm}$.

\section{Delineation, comparison and matching of GTV}

The images obtained by three scanning modes were processed by Tumor Loc Software under the same CT window setting (W: $1600 \mathrm{C:-600).} \mathrm{GTV}$ and $\mathrm{GTV}_{\mathrm{S}}$ were created from the two sets of CT images obtained by axial and spiral scanning modes, respectively (Figure $1 \mathrm{~A}$ and $\mathrm{B}$ ). The 3D coordinates of $\mathrm{GTV}_{\mathrm{A}}$ were $\mathrm{Ax}$, Ay and Az. The coordinates of $G_{T V}$ were Sx, Sy and Sz. calculating the distance from the centroidal position of $\mathrm{GTV}_{\mathrm{A}}$ and $\mathrm{GTV}_{\mathrm{S}}$ to the origin of coordinates, respectively. Tumor Loc software was used to retrospectively create the maximum intensity projection (MIP), and the ITVMIP was created from the MIP images [10] (Figure 1C). Using the Eclipse 8.6 treatment planning system, by which would conduct target registration on $\mathrm{GTV}_{\mathrm{A}}$ and $\mathrm{GTV}_{\mathrm{S}}$, then calculate the matching index (MI) between $\mathrm{GTV}_{\mathrm{A}}$ and $\mathrm{GTV}_{\mathrm{S}} \cdot \mathrm{MI}=\left(\mathrm{GTV}_{\mathrm{A}} \cap G T V_{\mathrm{S}}\right) /\left(\mathrm{GTV}_{\mathrm{A}} \cup G T V_{\mathrm{S}}\right)$ (nand $\cup$ were intersection and union, respectively) [11].

\section{Statistical analysis}

All statistical analyses were performed using the SPSS17.0 package (SPSS Inc, Chicago, IL, USA). The Wilcoxon test was used to compare the tumor volumes and the isocenter coordinates $(\mathrm{x}, \mathrm{y}, \mathrm{z}$ axial) gained by different scanning modes. A Pearson correlation analysis was utilized to study the relationship between MI and $\mathrm{GTV}_{\mathrm{S}}$. Differences were considered to be significant if the $p$-value was less than 0.05 .

\section{Results}

The average \pm standard deviation of $\mathrm{GTV}_{\mathrm{A}}$ and $\mathrm{GTV}_{\mathrm{S}}$ volumes were $9.94 \pm 9.74 \mathrm{~cm}^{3}$ (range $1.72 \sim 36.56 \mathrm{~cm}^{3}$ ) and $10.14 \pm 10.25 \mathrm{~cm}^{3}$ (range $1.45 \sim 35.41 \mathrm{~cm}^{3}$ ), respectively. There was no statistically significant difference between the volume of $\mathrm{GTV}_{\mathrm{A}}$ and $\mathrm{GTV}_{\mathrm{S}} \quad(p=0.16)$ (Table 1). The volume of ITV $\mathrm{V}_{\text {MIP }}$ was $14.33 \pm 12.47 \mathrm{~cm}^{3}$ (rang $2.93 \sim 41.16 \mathrm{~cm}^{3}$ ). On pair wise analysis using Wilcoxon rank test, ITV $\mathrm{IIP}_{\mathrm{M}}$ was significantly larger than $\operatorname{GTV}_{\mathrm{A}}$ and $\mathrm{GTV}_{\mathrm{S}}\left(p_{s}=0.000\right)$ (Table 1), and the ratios of $\mathrm{ITV}_{\mathrm{MIP}}$ to $\mathrm{GTV}_{\mathrm{A}}$ and $\mathrm{GTV}_{\mathrm{S}}$ were $1.57 \pm 0.54$ and $1.66 \pm$ 0.61 , respectively.

The center of tumor coordinates for $\mathrm{GTV}_{\mathrm{A}}, \mathrm{GTV}_{\mathrm{S}}$ and ITV $_{\text {MIP }}$ were listed in Table 2 and the averages \pm standard deviations were showed in Table 3. No differences were determined in $\mathrm{x}, \mathrm{y}$ and $\mathrm{z}$ directions $\left(p_{\mathrm{x}}=0.17\right.$, $\left.p_{\mathrm{y}}=0.40, p_{z}=0.48\right)$. The distances from the center of $\mathrm{GTV}_{\mathrm{A}}$ and $\mathrm{GTV}_{\mathrm{S}}$ to the origin of coordinate were $36.94 \pm$ $24.64 \mathrm{~cm}$ and $36.88 \pm 24.39 \mathrm{~cm}$, respectively. There are no difference between the two distances $(p=0.51)$.

MI between $G_{T V}$ and $G_{T V}$ was $0.41 \pm 0.24$; the variation range was from 0 to 0.89 . The correlation between the MI and $G_{T V}$ was illustrated in Figure 2. There was a positive correlation between MI and $\mathrm{GTV}_{\mathrm{S}}$. The correlation coefficients was $(r=0.64 p=0.002)$. 


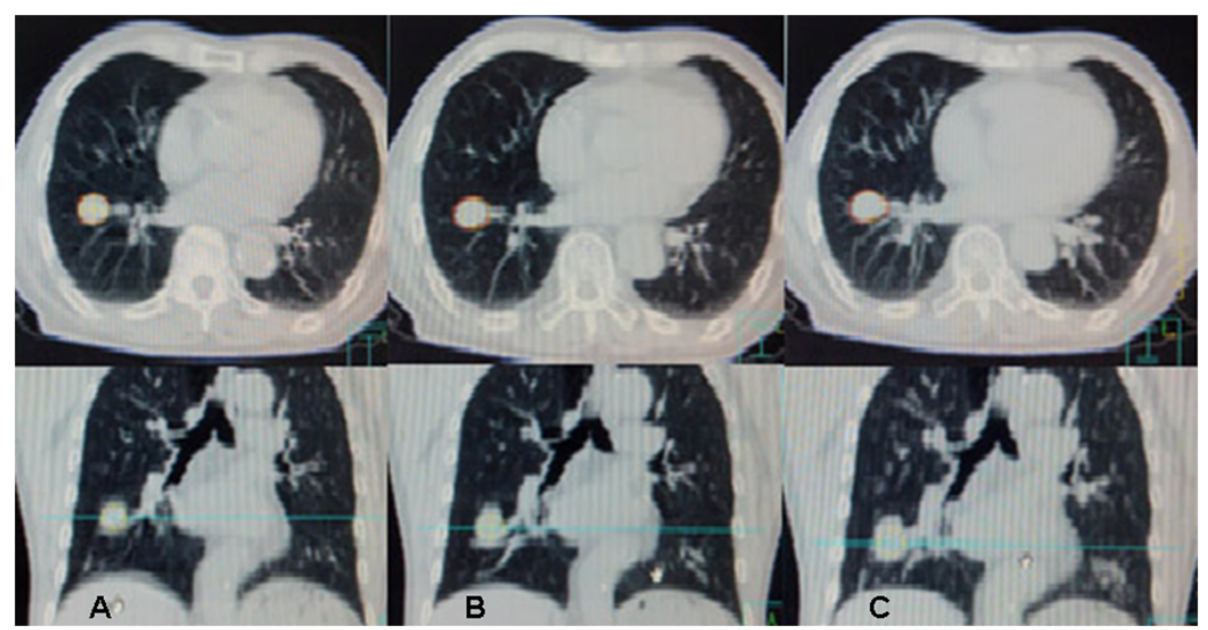

Figure $1 \mathrm{GTV}_{\mathbf{A}}, \mathrm{GTV}_{\mathbf{S}}$ and $\mathrm{ITV}_{\text {MIP }}$ were created from the three sets images. A: axial scan, B: spiral scan, $\mathbf{C}: 4 \mathrm{D}$ scan, respectively. And the coronal sections were reconstructed at the same time.

\section{Discussion}

SBRT is a safe and effective alternative therapy for patients with the inoperable lung cancer or metastatic tumor [12]. Physiologic motions such as respiratory motion and heart beat would lead to tumor displacement and change in shape, so delineation of the GTV was a critical component in the radiotherapy process for thoracic tumor [2]. Investigators have determined the target margin of thoracic tumor using the technology of active breathing control and 4DCT, which could reduce the interference on radiotherapy and increase the irradiation dose in target margin [13-16].

4DCT allowed for the clear delineation of the anatomical structures during free- breathing. This technique is widely used in evaluating the displacement of tumor and organ for thoracic and abdominal cancers. The ten sequence images reconstructed by $4 \mathrm{D}$ volume data could demonstrate movements of the tumor dynamically, and the $\mathrm{ITV}_{\mathrm{MIP}}$ represented the volume where tumor was present at any time within the respiratory cycle, which contained motion information of the tumor [9,17-19]. The axial scanning mode was scanning alternates with the movement of scanner couch, that was, "scanning-in couch-scanning" pattern. As a result, the simulation time was long. Taking the ordinary 16 layers CT for example, the $\mathrm{X}$-ray tube needs $1 \mathrm{~s}$ to rotate 360 degree and the scan cycle was $2.8 \mathrm{~s}$ by the axial mode, the

Table 1 Statistical comparison of tumor volume according different scanning modes

\begin{tabular}{lr}
\hline Comparison & $\boldsymbol{p}$-value \\
\hline GTV $_{\text {A VS GTV }}$ & 0.159 \\
ITV $_{\text {MIP VS GTV }}$ & 0.000 \\
ITV $_{\text {MIP VS GTV }}$ & 0.000 \\
\hline
\end{tabular}

scanning range was $24 \mathrm{~mm}$ (with $16 \times 1.5 \mathrm{~mm}$ detector). The spiral mode was continuous scanning with CT scanner couch moving at a speed of $30 \mathrm{~mm} / \mathrm{s}$. There was no interval during the scanning process. It takes only $0.75 \mathrm{~s}$ to complete the scan rang of $24 \mathrm{~mm}$ in superior-inferior direction. In this study, we recorded and compared the scanning time for the same range by axial and spiral mode, respectively. The result revealed that axial mode took a longer time to complete the same scan than spiral mode, $\mathrm{T}_{\mathrm{A}}=28.93 \pm 4.63 \mathrm{~s}, \mathrm{~T}_{\mathrm{S}}=8.81 \pm 1.41 \mathrm{~s}(p=0.000)$. Furthermore, the spiral mode could reduce motion artifacts, and increase the accuracy of tumor shape and position. Though the high speed of spiral mode, the scanning time for region of interest (ROI) was short for the small lesions and GTV contains little amount of respiratory motion information. The respiratory rate of the patients enrolled in the study was $16.00 \pm 2.81$ breaths/ $\mathrm{min}$, and the respiratory cycle was $3.87 \pm 0.73 \mathrm{~s}$. The axial and spiral scans often complete ROI within $1 \mathrm{~s}$. The key point was the ratio of the scanning time for ROI to the respiratory cycle. The higher the ratio was, the more motion information was included in the images. Among our subjects, there were 14 patients whose $\mathrm{GTV}_{\mathrm{A}}$ was larger than $\mathrm{GTV}_{\mathrm{S}}$, while 7 smaller. There was no statistically significant difference in volume between the two scanning modes. We analyzed the tumor motion information included in $\mathrm{GTV}_{\mathrm{A}}$ and $\mathrm{GTV}_{\mathrm{S}}$ by comparing the volumes of ITV $V_{\text {MIP }}$ with $G_{T V}$ and $G T V_{S}$, respectively. The ratio between $\operatorname{ITV}_{\mathrm{MIP}}$ and $\mathrm{GTV}_{\mathrm{A}}$ was $1.57 \pm$ $0.54(p=0.000)$. It suggested that the tumor motion information included in $\mathrm{GTV}_{\mathrm{A}}$ was limited. The result was similar to the reports of Nakamura M [20,21]. There was statistically significant difference in volume between $\operatorname{ITV}_{\text {MIP }}$ and $\operatorname{GTV}_{S}(p=0.000)$. The ratio between $\operatorname{ITV}_{\text {MIP }}$ and $G_{T V}$ was $1.66 \pm 0.61$, which suggested that the 
Table 2 The center of tumor coordinates for GTV $_{A}$, GTV $_{S}$ and ITV

\begin{tabular}{|c|c|c|c|c|c|c|c|c|c|}
\hline \multirow[t]{2}{*}{ case } & \multicolumn{3}{|c|}{$\mathrm{GTV}_{\mathrm{A}}$} & \multicolumn{3}{|c|}{$\mathrm{GTV}_{\mathrm{S}}$} & \multicolumn{3}{|c|}{ ITV $_{\text {MIP }}$} \\
\hline & $x$ & $y$ & $z$ & $x$ & $y$ & $z$ & $x$ & $y$ & $z$ \\
\hline 1 & -7.69 & -71.84 & -8.5 & -7.56 & -70.14 & -8.33 & -7.62 & -70.89 & -8.67 \\
\hline 2 & -7.46 & -70.79 & -4.92 & -7.67 & -69.39 & -5.02 & -7.68 & -69.84 & -4.81 \\
\hline 3 & 7.89 & -76.79 & -8.03 & 7.89 & -77.34 & -7.78 & 7.85 & -77.34 & -8.03 \\
\hline 4 & -4.49 & -57.69 & -0.74 & -4.44 & -57.89 & -0.56 & -4.45 & -57.84 & -0.73 \\
\hline 5 & -7.58 & -57.59 & 0.72 & -7.41 & -57.79 & 0.72 & -7.49 & -57.69 & 0.86 \\
\hline 6 & -2.8 & -49.44 & -5.92 & -2.75 & -49.44 & -5.75 & -3.02 & -49.34 & -5.88 \\
\hline 7 & -7.21 & -66.69 & -10.81 & -7.07 & -65.89 & -10.91 & -7.17 & -66.04 & -10.61 \\
\hline 8 & 3.64 & 7.51 & -0.52 & 3.49 & 7.86 & -0.33 & 3.69 & 7.66 & -0.67 \\
\hline 9 & -9.93 & -33.24 & 2.17 & -10.14 & -33.39 & 2.12 & -10.26 & -33.24 & 2.34 \\
\hline 10 & 8.52 & -24.84 & -5.35 & 8.88 & -25.29 & -5.18 & 8.5 & -24.64 & -5.33 \\
\hline 11 & -3.41 & -18.39 & 5.74 & -3.33 & -18.69 & 5.96 & -3.59 & -18.64 & 5.66 \\
\hline 12 & -9.57 & -10.49 & -9.92 & -9.57 & -10.74 & -9.71 & -9.59 & -10.79 & -9.93 \\
\hline 13 & 5.8 & -4.29 & -3.36 & 6.07 & -4.39 & -3.52 & 5.71 & -4.3 & -3.44 \\
\hline 14 & 10.47 & -8.69 & 0.59 & 10.4 & -8.24 & 0.97 & 10.48 & -8.69 & 0.74 \\
\hline 15 & -4.01 & -16.59 & -7.27 & -3.94 & -16.89 & -7.08 & -4.15 & -15.39 & -7.37 \\
\hline 16 & -8.35 & -3.59 & -5.29 & -8.38 & -4.09 & -5.43 & -8.08 & -4.04 & -5.4 \\
\hline 17 & 9.75 & -50.69 & -5.45 & 9.86 & -51.09 & -5.01 & 9.93 & -51.59 & -5.02 \\
\hline 18 & -5.83 & -59.24 & -3.81 & -5.94 & -59.19 & -3.71 & -5.81 & -59.34 & -4.00 \\
\hline 19 & 5.62 & 18.86 & -3.1 & 5.28 & 18.36 & -3.67 & 5.55 & 18.51 & -3.32 \\
\hline 20 & -5.41 & -9.56 & -1.86 & -5.31 & -9.41 & -1.95 & -5.49 & -9.96 & -2.07 \\
\hline 21 & -4.49 & -18.39 & -7.18 & -4.57 & -18.64 & -7.31 & -4.62 & -18.34 & -7.16 \\
\hline
\end{tabular}

motion information included in $\mathrm{GTV}_{\mathrm{S}}$ by spiral mode was limited too. The result of this study revealed that there was no difference in the volume of GTV by axial or spiral mode in 3DCT simulation. The tumor was moving when the axial and spiral scanning was performed during freebreathing. The $G_{T V} V_{3 D}\left(G T V_{A}\right.$ and $\left.G T V_{S}\right)$ created from the axial and spiral images included little respirationinduced motion information. It only represented the instant shape and volume that comes randomly by axial or spiral mode during the respiratory cycle.

ITV $_{\text {MIP }}$ was significantly larger than $G_{T V}$ and $G T V_{S}$. The differences in volume between $\mathrm{GTV}_{3 \mathrm{D}}$ and $\mathrm{ITV}_{\mathrm{MIP}}$ were caused by the tumor motion in three dimensions. The motion information was included in ITV $_{\text {MIP. }}$. The motion amplitude in superior-inferior direction was

Table 3 The comparison of 3D coordinates for GTV $_{A}$, GTV $_{S}$ and ITV MIP $(\bar{x} \pm s)$

\begin{tabular}{cccc}
\hline Scan modes & $\mathbf{x}$ axial & y axial & $\mathbf{z}$ axial \\
\hline $\mathrm{GTV}_{\mathrm{A}}$ & $-1.74 \pm 6.99$ & $-32.50 \pm 29.07$ & $-3.94 \pm 4.20$ \\
$\mathrm{GTV}_{S}$ & $-1.72 \pm 7.02$ & $-32.46 \pm 28.83$ & $-3.88 \pm 4.22$ \\
$\mathrm{IV}_{\mathrm{MIP}}$ & $-1.78 \pm 7.02$ & $-32.47 \pm 28.95$ & $-3.94 \pm 4.20$ \\
$x^{2}$ & 3.51 & 1.83 & 1.49 \\
$p$ & 0.17 & 0.40 & 0.48 \\
\hline
\end{tabular}

larger than those in lateral and anterior-posterior directions. The superior-inferior motion of GTV centroid in lower lobe was larger than the in upper lobe $[22,23]$. So the internal target volume (ITV) was generated from $\mathrm{GTV}_{3 \mathrm{D}}$ by adding different margins in $\mathrm{x}, \mathrm{y}$ and $\mathrm{z}$ directions. The compensated volume was still larger than ITV $_{\text {MIP }}$ created by $4 \mathrm{D}$ images.

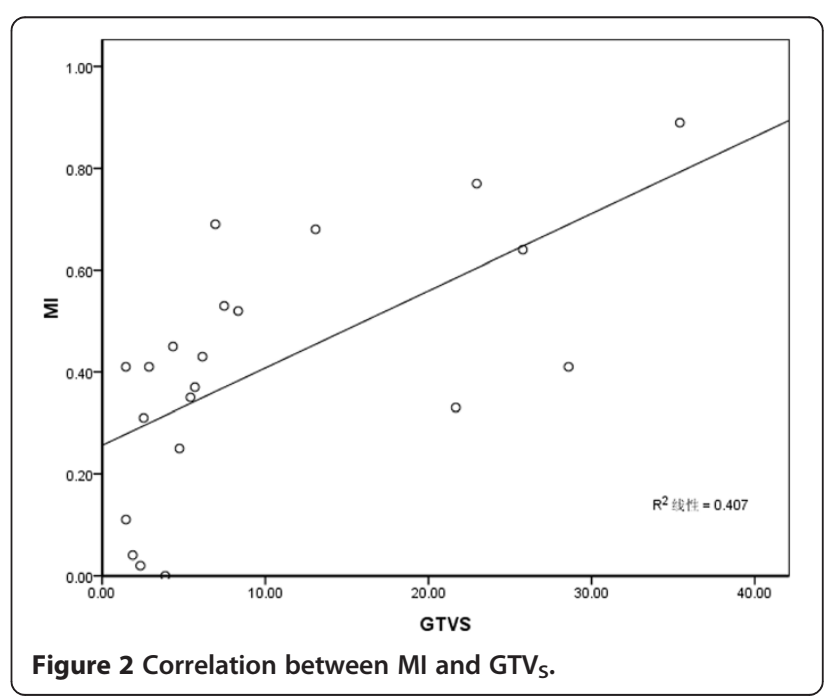


The axial and spiral scan were all performed during free-breathing. The centroid coordinates of $\mathrm{GTV}_{\mathrm{A}}$ and $\mathrm{GTV}_{\mathrm{S}}$ were instant location during the respiratory cycle. There was no statistically significant difference in 3D coordinates of $\mathrm{GTV}_{\mathrm{A}}$ and $\mathrm{GTV}_{\mathrm{S}}$, nor the distances from the centroidal positions to origin of coordinates. What's more, there was no difference in the centroidal position between $\mathrm{GTV}_{3 \mathrm{D}}$ and ITV $\mathrm{ITIP}_{\mathrm{M}}\left(p_{\mathrm{x}}=0.17, p_{\mathrm{y}}=0.40, p_{z}=0.48\right)$. This suggests that there was no obvious impact on for the tumors' central position by the three scanning modes during free-breathing.

Although there was no difference in volume and the centroidal coordinates between the axial and spiral modes during free-breathing, there was still respirationinduced change in shape of tumor [24,25]. We conducted target registration on $\mathrm{GTV}_{\mathrm{A}}$ and $\mathrm{GTV}_{\mathrm{S}}$, MI range from 0 to 0.89 . If the two target regions coincide completely, MI was 1 , otherwise, that was 0 . There was one case with no coincidence in our data, which suggested an obvious tumor displacement, and/or the tumor shapes varied greatly. Furthermore, there was a linear correlation between $\mathrm{MI}$ and $\mathrm{GTV}_{\mathrm{S}}(r=0.64, p=0.002)$. The small volume of GTV was one of the reasons for MI declination. The variation of MI was large in individuals. The low MI didn't mean increase the margins, and different MI didn't mean asymmetric margin either, because MI wasn't determined by the displacement and the change in shape, it was affected by the tumor volume also.

\section{Conclusion}

In summary, our study found no impact on the volume and centroidal position of GTV by the axial or spiral scan in 3DCT simulation. There were no difference in the centroidal positions between 3DCT and 4DCT as well. The tumor motion information included in $\mathrm{GTV}_{\mathrm{A}}$ and $\mathrm{GTV}_{\mathrm{S}}$ was both limited. A linear correlation was found between $\mathrm{MI}$ and $\mathrm{GTV}_{\mathrm{S}}$. Relative to axial scanning mode, spiral scan was more timesaving, reduced some motion artifacts and increased the accuracy of GTV due to its high scanning speed. So the spiral mode was a reasonable option to replace axial scan in 3DCT simulation for SPL.

\footnotetext{
Abbreviations

CT: Computed tomography; 3DCT: Three-dimensional CT; SPL: Solitary pulmonary lesion; 4DCT: Four-dimensional CT; 3D-CRT: Three-dimensional conformal radiotherapy; GTV: Gross tumor volume; GTV : Gross tumor volume gained by axial scan; $T_{\mathrm{S}}$ : Gross tumor volume gained by spiral scan; ITV MIP: Internal target volume gained by 4D maximum intensity projection; MIP: Maximum intensity projection; SBRT: Stereotactic body radiotherapy; RPM: Real-Time Position Management.; MI: Matching index; $\mathrm{T}_{\mathrm{A}}$ : Time of completing the scan by axial mode; $\mathrm{T}_{\mathrm{S}}$ : Time of completing the scan by spiral mode; ROI: Region of interest; $\mathrm{GTV}_{3 D}$ : $\mathrm{GTV}_{\mathrm{A}}$ and $\mathrm{GTV}_{\mathrm{S}}$; ITV: Internal target volume.
}

\section{Competing interests}

The authors declare that they have no competing interests.

\section{Authors' contributions}

D-PS delineated GTV in different series CT images and performed the statistical analysis. CL collected the CT images. YY conceived of the study, and helped to draft the manuscript. All authors read and approved the final manuscript.

\section{Acknowledgement}

This study was supported in part by Shandong Province Science and Technology Development Project (2012YD18089). Many colleagues helped to collect and analyze data. Their support and helps should be appreciated.

Received: 27 November 2013 Accepted: 28 August 2014

Published: 12 November 2014

\section{References}

1. Nakayama $H$, Satoh $H$, Kurishima K, Ishikawa $H$, Tokuuye K: High-does conformal radiotherapy for patients with stage III non-small-cell lung carcinoma. Int J Radiat Oncol Biol Phys 2010, 78:645-650.

2. Bradley JD, Nofal AN, El Naqa IM, Lu W, Liu J, Hubenschmidt J, Low DA, Drzymala RE, Khullar D: Comparison of helical, maximum intensityprojection (MIP), and averaged intensity (AI) 4D CT imaging for stereotactic body radiation therapy (SBRT) planning in lung cancer. Radio Oncol 2006, 81:264-268.

3. Pan T, Lee TY, Rietzel E, Chen GT: 4D-CT imaging of a volume influenced by respiratory motion on multi-slice CT. Med Phys 2004, 31:333-340.

4. Vedam SS, Keall PJ, Kini VR, Mostafavi H, Shukla HP, Mohan R: Acquiring a four dimensional computed tomography dataset using an external respiratory signal. Phys Med Biol 2003, 48:45-62.

5. Ford EC, Mageras GS, Yorke E, Ling CC: Respiration-correlated spiral CT: a method of measuring respiratory-induced anatomic motion for radiation treatment planning. Med Phys 2003, 30:88-97.

6. Xianfu L, Bangxian T, Mi L, Yujun L, Yeqin Z, Jin H, Daiyuan M: Impact of CT scanning condition on the precision and accuracy of target position. China J Radiat Oncol 2009, 18:142-145.

7. Wei H, Zheng F, Min F, Tonghai L, Heyi G, Baosheng L: Comparison of three CT scan methods used in precise radiotherapy of non-small cell lung cancer. China J Radiol Med Prot 2009, 29:65-67.

8. Dunn L, Kron T, Tayor ML, Callahan J, Franich RD: A phantom for testing of 4D-CT for radiotherapy of small lesions. Med. Phys 2012, 39:5372-5383.

9. Cole AJ, O'Hare JM, McMahon SJ, McGarry CK, Butterworth KT, McAleese J, Jain S, Hounsell AR, Prise KM, Hanna GG, O'Sullivan JM: Investigating the potential impact of four-dimensional computed tomggraphy (4DCT) on toxicity, outcomes and dose escalation for radical lung cancer radiotherapy. Clin Oncol 2014, 26:142-150.

10. Underberg RW, Lagenwaard FJ, Slotman BJ, Cuijpers JP, Senan S: Use of maximum intensity projections (MIP) for target volume generation in 4DCT scans for lung cancer. Int J Radiat Oncol Biol Phy 2005, 63:253-260.

11. Ezhil M, Vedam S, Balter P, Choi B, Mirkovic D, Starkschall G, Chang JY: Determination of patient-specific internal gross tumor volumes for lung cancer using four-dimensional computed tomography. Radia Oncol 2009, 4:1-14.

12. McGarry RC, Papies $L$, Williams R, Williams M, Whitford T, Timmerman RD: Stereotactic body radiation therapy of early-stage non-small-cell lung carcinoma: phase I study. Int J Radiat Oncol Biol Phys 2005, 63:1010-1015.

13. Bosmans G, Van Baardwijk A, Dekker A, Ollers M, Boersma L, Minken A, Lambin P, De Ruysscher D: Intra-patient variability of rumor volume and tumor motion during conventionally fractionated radiotherapy for locally advanced on-small-cell lung cancer:a prospective clinical study. Int J Radiat Oncol Biol Phys 2006, 66:748-753.

14. Yonghua $Y$, Yufen W, Shoufang G, Renben W, Wenwu L, Baosheng L: The influence on the position of peripheral lung cancer by the respiratory movement and the establishment of the mathematical model. China J Radiat Oncol 2004, 13:83-85.

15. Cheung PC, Sixel KE, Tirona R, Along YC: Reproducibility of lung tumor position and reduction of lung mass within the planning target volume using active breathing control (ABC). Int J Radiat Oncol Biol Phys 2003, 57:1437-1442

16. Wang L, Hayes S, Paskalev K, Jin L, Buyyounouski MK, Ma CC, Feigenberg S: Dosimetric comparison of stereotactic body radiotherapy using 4D CT and multiphase $\mathrm{CT}$ images for treatment planning of lung cancer: 
eevaluation of the impact on daily dose coverage. Radiother Oncol 2009, 91:314-324

17. Low D: $4 D$ imaging and $4 D$ radiation therapy: a new era of therapy design and delivery. Front Radiat Ther Oncol 2011, 43:99-117.

18. Li FX, Li JB, Zhang YJ, Liu TH, Tian SY, Xu M, Shang DP, Ma CS: Comparison of the planning target volume based on three dimensional $\mathrm{CT}$ and four dimensional CT images of non-small-cell lung cancer. Radio Oncol 2011, 99:176-180.

19. $Y u$ ZH, Lin SH, Balter $P$, Zhang L, Dong L: A comparison of tumor motion characteristics between early stage and locally advanced stage lung cancers. Radiother Oncol 2012, 104:33-38.

20. Dongping S, Minghuan L, Jianbin L, Yong Y, Jinming Y, Jun D: The application of four-dimensional $\mathrm{CT}$ technique in the planning target volume of the solitary pulmonary lesion. China J Radiat Oncol 2011, 20:417-419.

21. Nakamura M, Narita Y, Matsuo Y, Narabayashi M, Nakata M, Yano S, Miyabe Y, Matsugi K, Sawada A, Norihisa Y, Mizowaki T: Geometrical differences in target volume between slow $\mathrm{CT}$ and $4 \mathrm{D} \mathrm{CT}$ imaging in stereotactic body radiotherapy for lung tumors in the upper and middle lobe. Med Phy 2008, 35:4142-4148.

22. Hof H, Rhein B, Haering P, Kopp-Schneider A, Debus J, Herfarth K: 4D CT based target volume definition in stereoactic radiotherapy of lung tumors:comparison with a convertional technique using individual margins. Radiother Oncol 2009, 93:419-423.

23. Weiss E, Wijesooriya K, Dill SV, Keall PJ: Tumor and normal tissue motion in the thorax during respiration: analysis of volumetric and positional variations using 4DCT. Int J Radiat Oncol Biol Phys 2007, 67:296-307.

24. Adamson J, Zhuang T, Yin FF: Contour based respiratory motion analysis for free breathing CT. Comput Biol Med 2011, 41:908-915.

25. Hallman JL, Mori S, Sharp GC, Lu HM, Hong TS, Chen GT: A Four-dimensional computed tomography analysis of multiorgan abdominal motion. Int J Radiat Oncol Biol Phys 2012, 83:435-441.

doi:10.1186/1748-717X-9-211

Cite this article as: Shang et al:: A comparison of the different 3D CT scanning modes on the GTV delineation for the solitary pulmonary lesion. Radiation Oncology 2014 9:211.

\section{Submit your next manuscript to BioMed Central and take full advantage of:}

- Convenient online submission

- Thorough peer review

- No space constraints or color figure charges

- Immediate publication on acceptance

- Inclusion in PubMed, CAS, Scopus and Google Scholar

- Research which is freely available for redistribution 